\title{
QUANTIFYING CELL-ADHESION STRENGTH WITH MICROPIPETTE MANIPULATION: PRINCIPLE AND APPLICATION
}

\author{
Jin-Yu Shao, Gang Xu and Peng Guo
}

Department of Biomedical Engineering, Washington University, Saint Louis, MO 63130

\section{TABLE OF CONTENTS}

\section{Abstract}

2. Introduction

3. The step-pressure technique

4. The biomembrane-force probe

5. The micropipette-aspiration technique

6. Requirements of the MAT

7. Applications of the MAT

8. Concluding remarks

9. Acknowledgements

10. References

\section{ABSTRACT}

Quantifying cell-adhesion strength is of great importance in biology and medicine. Cell-adhesion strength can be characterized by separating two adherent cells and determining the force required to do so, or by measuring the lifetime of a receptor-ligand bond that mediates cell adhesion. To this end, several micropipette-based experimental techniques that operate at both cellular and molecular levels have been developed over the past few decades. In this review, we provide an overview of three of these techniques, i.e., the step-pressure technique (SPT), the biomembraneforce probe (BFP), and the micropipette-aspiration technique (MAT). More detailed discussion will be given about the requirements and applications of the MAT.

\section{INTRODUCTION}

Intercellular adhesion, which is essential for preserving and maintaining living tissue integrity and function, has a wide spectrum of binding strength. While strong cell adhesion is necessary in some cases such as tight intercellular junctions, only weak cell adhesion is present in some other cases such as leukocyte rolling on the endothelium. Therefore, quantifying cell-adhesion strength is of great interest to biophysicists and bioengineers. To this end, many experimental techniques have been developed. At the cellular level, these techniques include methods that are based on centrifugation (1), hydrodynamic shear (parallel, radial, and rotational) (2-4), and micropipette manipulation (5). At the molecular level, they include atomic force microscopy (6), the optical trap (7), the microneedle technique (8), the magnetic force apparatus (9), the biomembrane-force probe (BFP) (10), and the micropipette-aspiration technique (MAT) (11). Many reviews have been written in regard to atomic force microscopy and the optical trap. In this paper, we present an overview of the quantification of cell-adhesion strength by means of micropipette manipulation at both cellular and molecular levels.

The application of micropipette manipulation to single cell studies can be traced back to Mitchison and
Swann, who first developed and employed a micropipettebased elastimeter to determine the internal pressure and the membrane elastic modulus of unfertilized sea urchin eggs $(12,13)$. Ten years after this pioneering effort, Rand and Burton further refined the elastimeter and applied it to the study of the viscoelastic properties of the erythrocyte membrane $(14,15)$. In the 1970 s and 80 s, the micropipettemanipulation system was significantly improved at several laboratories and applied to a myriad of studies on the mechanics of erythrocytes, leukocytes, endothelial cells, and lipid vesicles or liposomes (16-20). In these early studies, either a point force or a suction pressure over a small area was imposed on a single cell or liposome surface. If a suction pressure was imposed, its correlation with the aspirated projection length of the cell or liposome inside the micropipette would be obtained; if a tensile point force was imposed, its correlation with the growth rate of a long thin membrane tube (a tether), which was extracted from the cell or liposome, would be obtained. With the aid of theoretical analysis, these experimental data yielded many valuable material constants of cells or cell membranes such as Young's modulus, shear viscosity, expansion modulus, bending stiffness, and elastic shear modulus. These studies have been summarized in three excellent reviews by Mohandas and Evans, Waugh and Hochmuth, and Hochmuth (21-23). In the 1990s, micropipette manipulation was further extended to many other studies such as the metastasis of tumor cells and the two-dimensional (2-D) kinetics of cell-cell interactions (24-26).

Quantification of cell-adhesion strength is one of many applications of micropipette manipulation. Specific cell adhesion in biology is mediated by receptor-ligand interactions and the like. Consequently, cell-adhesion strength is traditionally characterized by receptor-ligand binding affinity and avidity. One common approach to quantifying this strength is to impose a pulling force to rupture the adhesive contact (single or multiple bonds) between two opposing surfaces. Different indicators are obtained depending on different types of applied force. If 
(a)
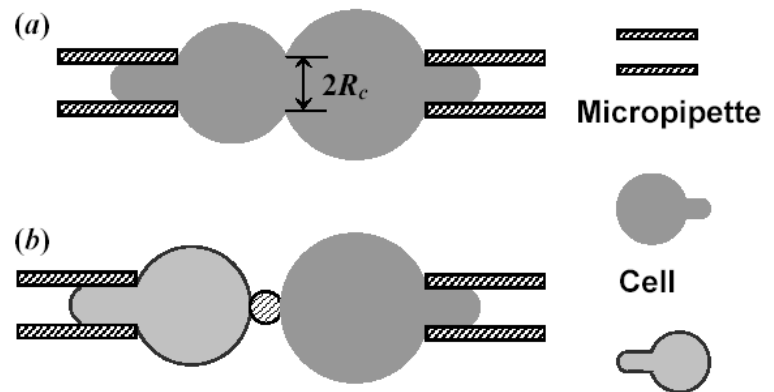

(c)

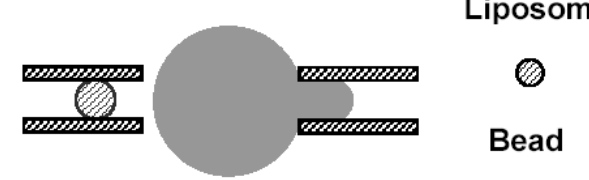

Figure 1. Schematic representations of three micropipettebased techniques for quantifying cell-adhesion strength: $a$ ) the step-pressure technique, $b$ ) the biomembrane-force probe, and $c$ ) the micropipette-aspiration technique. $R_{c}$ is the radius of the conjugation interface. All the cells on the right can be replaced with another type of object such as a bead or a flat substrate.

an increasing force is applied, the force magnitude at rupture will be measured; if a constant force is applied, the adhesion lifetime under this force will be measured. A variety of techniques are available for this purpose. Next, we will discuss three that are based upon a micropipettemanipulation system, namely the step-pressure technique (SPT), the BFP, and the MAT.

\section{THE STEP-PRESSURE TECHNIQUE (SPT)}

The step-pressure technique, which was developed by Sung et al. in 1986, is the first micropipettebased technique that was employed for quantifying celladhesion strength (17). The major components of a micropipette-manipulation system are micropipettes (cylindrical glass tubes with internal diameters of a few micrometers), micromanipulators (for controlling the positions of the micropipettes in an experimental chamber), and manometers (for controlling the pressures within the micropipettes). The resolution of the manometers typically used is about $0.01 \mathrm{pN} / \mu \mathrm{m}^{2}$ or $1 \mu \mathrm{m} \mathrm{H}_{2} \mathrm{O}(11,27)$.

Figure $1 a$ illustrates how the SPT works. One cell is held tightly on the right by a micropipette with a large suction pressure, while another is held on the left by another micropipette with a smaller suction pressure. First, the cell on the left is brought into contact with the cell on the right. These two cells are allowed to adhere to each other for some time. Then the cell on the left is pulled away. If adhesion has developed and the suction pressure in the left pipette is not large enough, the cell on the left will slip out of the left pipette. Afterwards, the suction pressure in the left pipette will be increased stepwise and the whole procedure will be repeated until the cell on the left is separated from the cell on the right. The minimum suction pressure that leads to the complete separation of these two cells is referred to as the critical separation pressure $\left(P_{c}\right)$.
Then the critical separation stress $\left(S_{c}\right)$, whose magnitude represents the adhesion strength, will be calculated with an empirical equation (17),

$S_{c}=2 P_{c}\left(\frac{R_{p}}{R_{c}}\right)^{2}$,

where $R_{p}$ is the radius of the left micropipette and $R_{c}$ is the radius of the conjugation interface (Figure 1a).

Sung et al. first applied the SPT to determining the critical separation stress of a cytotoxic $\mathrm{T}$ cell and one of its specific target cells, JY (a B lymphoblastoid cell line), and obtained a value of $1.5 \mathrm{nN} / \mu \mathrm{m}^{2}$ for $S_{c}$ (17). A biophysical model was later established for relating the adhesion energy density between these two cells, which is defined as the adhesion energy per unit conjugation area, to the instantaneous conjugation area during the separation process. This energy density was found to increase from $0.2 \mathrm{dyn} / \mathrm{cm}$ to $1.4 \mathrm{dyn} / \mathrm{cm}$ while the conjugation area decreased from $6.5 \mu \mathrm{m}^{2}$ to $0.5 \mu \mathrm{m}^{2}$. With this model, the authors also found that the reciprocal of the adhesion energy density is linearly proportional to the conjugation area (28). The second application of the SPT made by Sung et al. was to characterize the adhesion strength between HL-60 cells (model leukocytes) and IL-1 $\beta$-stimulated endothelial cells. However, they were able only to determine an average separation force of $744 \mu$ dyn because it was difficult to measure the conjugation area (29). These results showed that the SPT is reasonably effective in quantifying cell-adhesion strength on a cellular scale.

The cell on the right shown in Figure $1 a$ can be replaced by a protein-coated substrate (or any other surface such as a flat plate with cells in culture) so that cellsubstrate adhesion strength can be determined. For this type of experiment, a biophysical model was also established by assuming the cell on the left is a viscoelastic sphere (radius $R_{s}$ ) (30). With this model, the adhesion energy density between the cell and substrate $(\gamma)$ can be calculated with $\gamma=P_{c} R_{p}^{2} / 3 R_{s}$.

In this manner, Tozeren et al. calculated the adhesion energy density between phorbol 12-myristate 13-acetate (PMA)-stimulated T lymphocytes and ICAM-1-coated substrates. They obtained a value of about $0.15 \mathrm{dyn} / \mathrm{cm}$, two orders of magnitude larger than the one reported for the interaction between Jurkat T-cells and LFA-3-coated substrates $(0.0013 \mathrm{dyn} / \mathrm{cm})$, which is known to be weak $(30,31)$. This modified SPT has also been applied to fibroblasts and laminin- or fibronectin-coated substrates, where relative adhesion strengths have been reported (32, 33).

For the SPT to work, one of the key requirements is that nonspecific adhesion between the cell and pipette wall has to be much weaker than the overall adhesion strength against the external cell or substrate. Besides, a final clean separation of these two cells is desired. If tethers are formed between the cells during the separation process, the assessment of whether the adherent cell is completely separated from the other cell or substrate will become relatively arbitrary (17). On the analytical side, because the 
biophysical properties of the adherent cells could be drastically different for different cell types, tailored analysis might be needed for each specific study. One recent important finding that should be included in a future modeling effort is that, when pulled, the lifetime of a receptor-ligand bond will depend on both the magnitude and loading rate of the applied force, i.e., the rupture force of the bond will depend on how fast it is stressed. As a result, the critical separation stress will depend on how fast the cell on the left is pulled away. Nevertheless, compared with other techniques like centrifugation, the SPT does allow us to observe two adherent cells in action and obtain a quantitative measure of their adhesion strength through some simple analyses.

\section{THE BIOMEMBRANE-FORCE PROBE (BFP)}

The biomembrane-force probe was developed by Evans et al. in 1995 (10). As shown in Figure $1 b$, an inflated lipid vesicle (liposome) or erythrocyte is held by a micropipette with a suction pressure while a latex bead is tightly attached to the vesicle or erythrocyte as the force transducer. The bead is coated with proteins of interest so that it can interact with the cell on the right. The pulling or pushing force imposed on the bead will result in the membrane deformation, which is equivalent to the extension or compression of a spring. Therefore, the whole system functions like a spring and the spring constant $\left(k_{f}\right)$ can be calculated by (34)

$k_{f}=2 \pi T_{m} /\left[\ln \left(2 R_{0} / R_{p}\right)+\ln \left(2 R_{0} / R_{b}\right)\right]$,

where $T_{m}$ is the membrane tension of the vesicle or erythrocyte, $R_{b}, R_{p}$, and $R_{0}$ are respectively the radii of the bead, left pipette, and vesicle or erythrocyte membrane capsule. Since $T_{m}$ and the suction pressure in the left pipette are related by the Law of Laplace, $k_{f}$ can be easily adjusted in the range of $0.001 \mathrm{pN} / \mathrm{nm}$ to 10 $\mathrm{pN} / \mathrm{nm}$ by changing the suction pressure in the left pipette. Thus, the loading rate of the force is also adjustable. This is the major advantage that the BFP has over other techniques.

The BFP can be used in imposing forces from 0.5 $\mathrm{pN}$ to over $1000 \mathrm{pN}$ with a wide range of loading rates from $0.1 \mathrm{pN} / \mathrm{s}$ to $10^{5} \mathrm{pN} / \mathrm{s}$ (35). To impose a constant force, the BFP needs a feedback system that allows a set amount of membrane deformation. The displacement of the BFP force transducer can be tracked with reflection interference contrast at nanometer resolution. With this sub-piconewton force and nanometer displacement capability, the BFP has proven to be an elegant system for studying a receptorligand bond subjected to a pulling force (36-38). Most importantly, Evans et al. have shown theoretically and experimentally that the rupture force of a receptor-ligand bond depends on how fast the bond is pulled, i.e., the loading rate of the force (39-44). For detailed discussion of this technique and its application to quantifying celladhesion strength, see the review by Evans (44).

\section{THE MICROPIPETTE-ASPIRATION TECHNIQUE (MAT)}

Shortly after the BFP was invented, the micropipette-aspiration technique was developed by Shao and Hochmuth (11). While the BFP is based upon the theoretical principle of solid mechanics, the MAT is fluid mechanical in nature. In other words, the force on the transducer is imposed by viscous forces rather than elastic deformation. As shown in Figure 1c, a spherical object, either a cell or a bead, can serve as the transducer of the MAT. A small clearance between the bead-transducer and pipette wall is necessary to enable the transducer to move freely inside the micropipette. A positive pressure will allow the bead, which is coated with proteins of interest, to contact and adhere to the cell on the right. An ensuing constant suction pressure $(\Delta p)$ will impose a pulling force on the adhesive bond and rupture the adhesion. The magnitude of the force on the transducer can be calculated by (11)

$F=\pi R_{p}^{2} \Delta p\left(1-\frac{4}{3} \bar{\varepsilon}\right)\left(1-\frac{U_{t}}{U_{f}}\right)$,

where $U_{f}$ is the free-motion velocity of the transducer at $\Delta p, U_{t}$ is the velocity of the transducer after adhesion at the same $\Delta p$, and

$\bar{\varepsilon}=\varepsilon / R_{p}$,

where $\varepsilon$ is the minimum gap width between the beadtransducer and pipette wall. For small gaps, $4 \bar{\varepsilon} / 3$ becomes negligible. If the transducer is stationary after its adhesion to the cell surface $\left(U_{t}=0\right), F$ reduces to the product of the cross sectional area of the pipette and the suction pressure. If the transducer moves at its free-motion velocity, i.e., in the absence of adhesion $\left(U_{t}=U_{f}\right), F$ will be zero. As long as $U_{t}$ is a constant after adhesion, which was often the case in previous experiments (11), a constant force can be easily imposed with the MAT without any feedback system.

For any micropipette-manipulation system, the pressure-drop $(\Delta p)$ resolution is a constant, so the force resolution of the MAT depends on $R_{p}$. For a bead of $9 \mu \mathrm{m}$ in diameter, a theoretical resolution of $1 \mathrm{pN}$ can be calculated from Eq. (4), corresponding to a pressure resolution of $1 \mu \mathrm{m} \mathrm{H}_{2} \mathrm{O}$ or $0.01 \mathrm{pN} / \mu \mathrm{m}^{2}$. Although it is possible to extend the range of force that can be imposed with the MAT to sub-piconewtons (45), the smallest force that has ever been imposed with a $9-\mu \mathrm{m}$ bead to date was about $15 \mathrm{pN}$ (46). The smallest bead ever used in the MAT was around $3.2 \mu \mathrm{m}$ in diameter, which allowed us to impose forces of a few piconewtons (unpublished data). With the single particle tracking technique (47), the beadtransducer displacement of the MAT can be tracked with an accuracy of a few nanometers. While the low force sensitivity of the MAT is similar to that of the optical trap, its high force range is much greater. With the MAT, forces on the order of hundreds of nanonewtons can be imposed. In the next two sections, we will discuss the requirements and applications of the MAT.

\section{REQUIREMENTS OF THE MAT}

For the MAT to work well, at least three conditions have to be satisfied during every experiment: 1) the transducer bead moves approximately along the axis of the left pipette (Figure 1c), 2) $\varepsilon<<R_{p}$, and 3) the distance between the cell and left pipette is appropriate, i.e., neither 


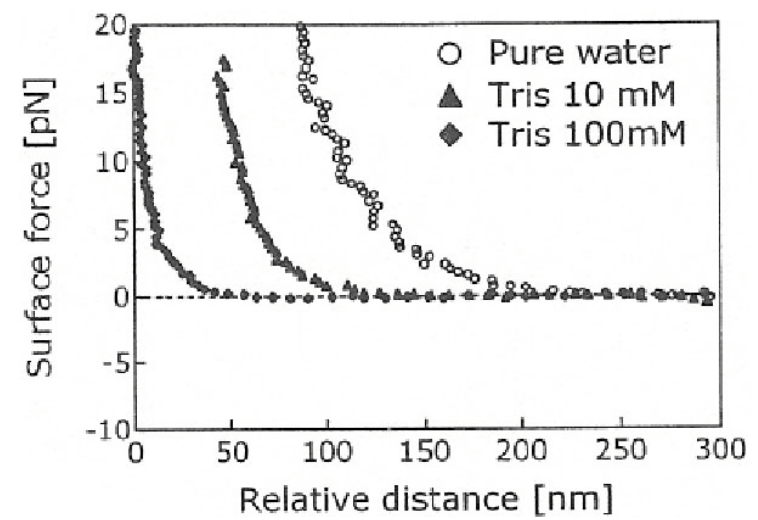

Figure 2. The repulsive force between a glass surface and a $1-\mu \mathrm{m}$ latex bead at different relative distances in pure water and two different buffers: Tris $10 \mathrm{mM}$ and $100 \mathrm{mM}$. From Ota (49).

too small nor too large. Since any adhesion between the bead-transducer and pipette wall will interfere with determination of the adhesion between the beadtransducer and the external cell on the right, it is critical to ensure that the bead does not adhere to the pipette wall.

Equation (4) is based on low Reynolds number hydrodynamics (creeping flow) under the assumption that the bead is exactly coaxial with the micropipette in which it moves. Accordingly, any spherical particle that is displaced from the tube axis will maintain its radial position because no hydrodynamic radial forces will be experienced by the sphere at zero Reynolds number (48). If the bead-transducer of the MAT drifts away from the tube axis, its free motion velocity $\left(U_{f}\right)$ could be quite different from the coaxial value even under the same suction pressure. However, the values of $U_{f}$ measured from all our experiments are quite consistent (unpublished data), indicating that the bead was indeed approximately coaxial to the micropipette. This fact can be explained by the existence of a centripetally-repulsive force exerted by the micropipette wall on the bead. This force, effective especially when $\varepsilon$ is small, inhibits the radially-outward drift of the bead. Figure 2 shows measurements of the repulsive force acting on a $1-\mu \mathrm{m}$ latex bead as it approached a glass surface in pure water and two different buffers (49). Obviously, this repulsive force could be very large (tens of piconewtons) when the distance between the two surfaces is small $(\leq$ about 100 $\mathrm{nm})$. Since the gap was approximately $100 \mathrm{~nm}$ in the MAT experiment where a latex bead was used as the force transducer (27), it is very likely that the surface force between the bead-transducer and pipette wall prevented the bead from drifting to an appreciably offaxis position. When a spherical neutrophil was used as the transducer, an equivalent gap width of $0.1 \mu \mathrm{m}$ was computed (11). Because the interaction force between a neutrophil and a glass surface has not been measured, it remains to be shown that a similar mechanism exists in this case. In addition to the repulsive force, hydrodynamic forces due to the squeezing film effect may also play a role in keeping the bead coaxial with the micropipette.

If the force transducer moves coaxially through the micropipette, the total pressure drop over the whole micropipette $(\Delta p)$ can be calculated by

$$
\begin{aligned}
& \Delta p=\frac{\mu U}{R_{p}}\left[\left(\frac{4 \sqrt{2} \pi}{\bar{\varepsilon}^{1 / 2}}-32\right)+8\left(\frac{L_{e q}}{R_{p}}-2(1-\bar{\varepsilon})\right)\left(1-\frac{4}{3} \bar{\varepsilon}\right)\right] \\
& +\frac{F}{\pi R_{p}^{2}}\left[\left(1+\frac{4}{3} \bar{\varepsilon}\right)+8\left(\frac{L_{e q}}{R_{p}}-2(1-\bar{\varepsilon})\right)\left(\frac{2 \sqrt{2}}{9 \pi} \bar{\varepsilon}^{5 / 2}\right)\right]
\end{aligned}
$$

where $R_{p}$ is the radius of the micropipette lumen, $\mu$ is the medium viscosity, $U$ is the velocity of the transducer with or without adhesion to a cell or substrate, and $L_{e q}$ is the equivalent length of the micropipette, which is defined as

$L_{e q}=\frac{\pi R_{p}^{4} \Delta p}{8 \mu Q}$

where $Q$ is the volumetric flow rate under $\Delta p$ when the bead-transducer is absent in the micropipette. Equation (6) is a more accurate description of the pressure drop than the equation derived earlier by Shao and Hochmuth (11). In the latter derivation, an arctangent term was approximated by $\pi / 2$, which resulted an error of about $10 \%$ if the force is directly calculated from the volumetric flow rate in the micropipette (unpublished data). For Eq. (4) to work, the last term in the second square bracket in Eq. (6) has to be small enough to be neglected, which requires that $\varepsilon<<R_{p}$ and that the equivalent length of the micropipette is not too long. If these conditions are not satisfied, the force calculation will become very complicated and that makes the MAT difficult to use. Besides, if $\varepsilon$ is not small enough for the repulsive force to have any effect, the bead-transducer may drift away from the micropipette axis and Eq. (6), on which Eq. (4) is based, may lose its validity. Large $\varepsilon$ will also have some adverse effects on the study of tether formation, which will be discussed in the next section.

The presence of the external cell on the right in Figure $1 c$, as long as it is outside the left pipette, does not contribute much to the resistance of the flow in the left micropipette. This was confirmed experimentally by Shao and Hochmuth (11) and again numerically by us (data not shown). Our numerical simulation also showed that, only when the shortest distance between the left pipette and the cell on the right becomes comparable to $\varepsilon$, the cell presence would have a large impact on the force calculation. However, the distance between the left pipette opening and the external cell on the right must not be too large either because most of the bead volume should remain inside the left pipette when $F$ is imposed. If the external cell in Figure $1 c$ is replaced by a much larger bead as in the study by Levin et al. (50) or a large flat substrate, a similar criterion can be applied. Figure 3 shows the mean velocity of the fluid in the left pipette when a semi-infinite plane replaces the external cell in Figure $1 c$ and is placed at different distances from the left pipette $(d)$. It is obvious that, as long as the distance between the left pipette and substrate is larger than $1 \mu \mathrm{m}$, its influence on the fluid velocity will be negligible. This indicates that, under this condition, the 


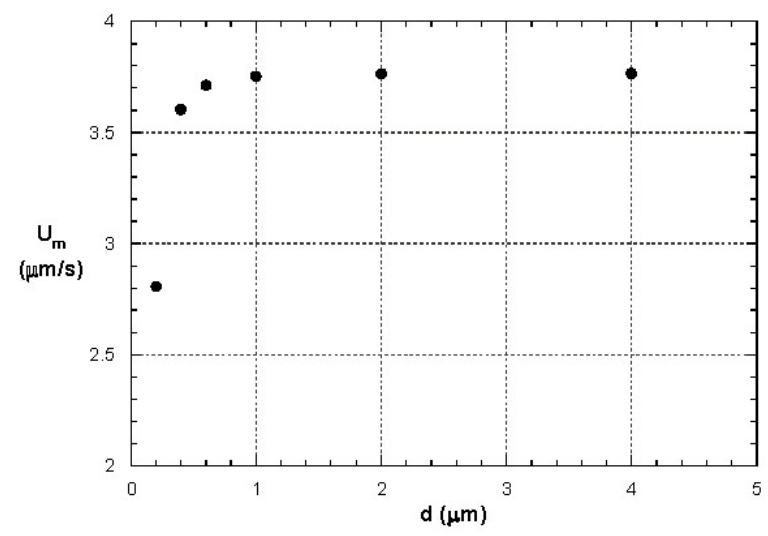

Figure 3. The mean velocity of the fluid $\left(U_{m}\right)$ when the external cell on the right in Figure $1 c$ is replaced by a large flat plate, which is placed at different distances from the left pipette opening $(d)$. The velocities were computed with FIDAP, a fluid dynamics simulation software package (Fluent Incorporated, Lebanon, $\mathrm{NH}$ ), by assigning a constant pressure drop from the far field to the outlet of the left pipette. Other parameters of the simulation are: $R_{p}=$ $4.1 \mu \mathrm{m}, R_{b}=4 \mu \mathrm{m}$, and the pipette wall thickness is 3.9 $\mu \mathrm{m}$.

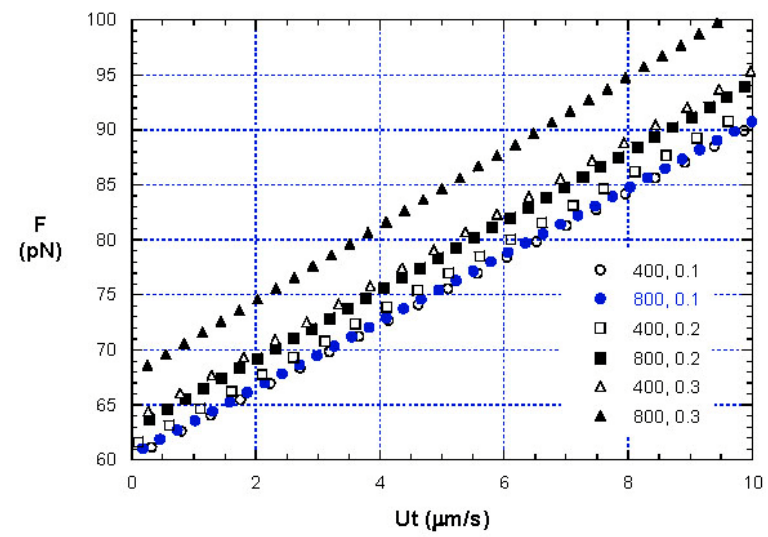

Figure 4. The pulling force calculated from Eq. (4) vs. the tether growth velocity calculated by combining Eq. (6) and an assumed tether force-velocity relationship of $F=60+$ $3 U_{t}$ (in $\mathrm{pN}$ ). Shown in the legends are $L_{e q}$ and $\varepsilon$, both in $\mu \mathrm{m}$. It is obvious that when $\varepsilon$ is large, Eq. (4) will overestimate the pulling force. If higher order terms of $\bar{\varepsilon}$ as shown in Eq. (6) are included in the force calculation, all the lines shown here will collapse into one that yields: $F=$ $60+3 U_{t}$ Other parameters used in the calculation are: $R_{p}=$ $4.6 \mu \mathrm{m}$ and $\mu=0.001 \mathrm{pN} \cdot \mathrm{s} / \mu \mathrm{m}^{2}$.

force calculation will be only weakly affected as well. The pipette wall thickness used in this simulation, $3.9 \mu \mathrm{m}$, is an overestimate. For thinner pipette walls, the data points shown in Figure 3 will shift to the left and the same criterion of $d>1 \mu \mathrm{m}$ will hold.

\section{APPLICATIONS OF THE MAT}

The MAT is very versatile and it has been applied to the study of: 1) membrane tether formation from single cells, 2) surface receptor expression, and 3) single molecule or bond mechanics. The first two are cellular level phenomena while the third is of molecular scale.

The MAT was first applied to studying the mechanics of membrane tethers extracted from passive human neutrophils (11). To extract a tether, a point force is applied by means of a receptor on a cell surface or a modified lipid molecule on a liposome. It has been shown theoretically and experimentally (also with other techniques such as the optical trap and other cell types such as activated neutrophils, endothelial cells, and lymphocytes $(27,51,52)$ that a linear relationship exists between the pulling force $(F)$ and tether growth velocity $\left(U_{t}\right)$,

$$
F=F_{0}+2 \pi \mu_{e f f} U_{t}=F_{0}+2 \pi \mu_{\text {eff }} \frac{d L}{d t}
$$

where $L$ is the tether length, $F_{0}$ is the threshold force that is determined by the membrane tension, bending stiffness, and adhesion energy between the membrane and cytoskeleton, and $\mu_{\text {eff }}$ is the effective viscosity that is determined by the membrane viscosity, interbilayer slip, and membrane slip over the cytoskeleton $(11,51,53-56)$. For cells with excess membrane surface area, the adhesion energy between the membrane and cytoskeleton can be calculated from $F_{0}$ (57). According to Eq. (8), the slope $d F / d U_{t}>0$. However, according to Eq. (4), $d F / d U_{t}<0$. This shows that, for any $R_{p}$ and $\Delta p$, cell heterogeneity will cause the data obtained with the MAT to scatter in a direction that crosses the intended $F-U_{t}$ correlation shown in Eq. (8). A simple error analysis will also show that larger scatter is expected at larger pressures. To eliminate this type of scatter, we need to average all the measurements obtained at the same $\Delta p$. This way of treating the data is labor-intensive because a lot of data points must be generated at each $\Delta p$, but it is very effective even at different equivalent lengths as long as $\varepsilon$ is small, as shown in Figure 4. Figure 4 also shows that it is essential to have a small $\varepsilon$. Otherwise, erroneous correlations between $F$ and $U_{t}$ would be obtained with Eq. (4).

The MAT can also be used in studying twodimensional receptor-ligand kinetics. In this regard, it is similar to the technique developed by Chesla et al. (24). The difference between these two techniques depends on how adhesion events are determined. While the technique developed by Chesla et al. employs observable cellular deformation as an indicator for adhesion events, the MAT detects transducer velocity changes for the same purpose. With the MAT, the contact area between the beadtransducer and cell is controlled with a well-defined positive pressure (27). For short contact time, this type of control can create fairly consistent contact areas. For long contact time, the contact area may increase over time due to the viscoelastic properties of the cell. At constant contact area and contact time, the change in the receptor expression or affinity can be inferred from the change in the adhesion frequency, which is the total number of adhesion events divided by the total number of contacts. When combined with the kinetic analysis for small systems, the adhesion frequency will yield the forward and reverse reaction rate constants. With the MAT, Levin et al. successfully studied the expression of E-selectin on endothelial cells after IL- $1 \alpha$ 


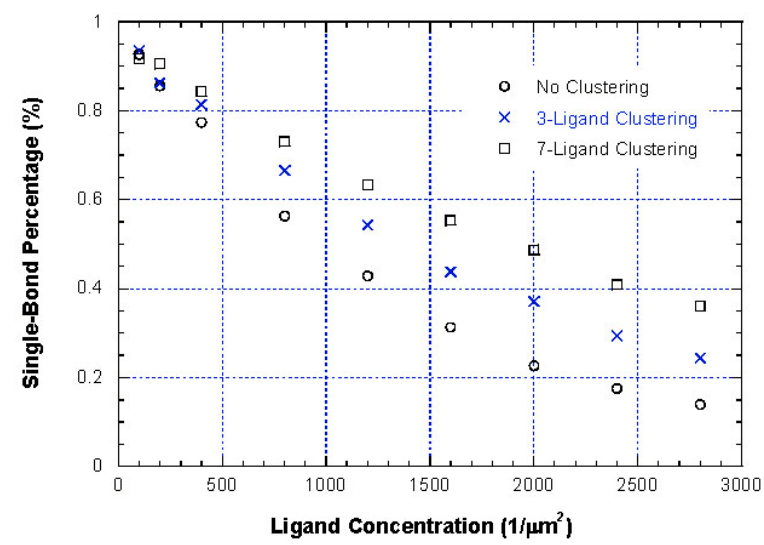

Figure 5. Dependence of the single-bond percentage among all adhesion events on the ligand concentration in one hundred contacts between two interacting surfaces (a cell and a ligand-coated bead) for three cases: $a$ ) no ligand clustering, $b$ ) three-ligand clustering, and $c$ ) seven-ligand clustering.

stimulation, which increased from 0 to $742 / \mu \mathrm{m}^{2}$ in five hours (50). The two-dimensional forward rate constant was calculated to be about $3.7 \times 10^{-6} \mu \mathrm{m}^{2} / \mathrm{s}$, which is in the same neighborhood as that of other similar interactions $(24,58)$.

Compared with other techniques, the strength of the MAT is in its ability to study cell-cell interactions directly because a spherical cell can be employed as the transducer. One example is that, after $\beta_{2}$ integrins are induced to their high- or low-affinity forms by cations (magnesium, manganese, and calcium), human neutrophils remain spherical and can be used directly as the force transducer of the MAT. Spillmann et al. took advantage of this feature and investigated the influence of divalent cations on neutrophil homotypic adhesion (59), while Lomakina and Waugh studied the interaction between neutrophils and ICAM-1-coated substrates (60).

As in tether extraction, a constant point force can be imposed with the MAT on a single molecular bond between two opposing surfaces. Thus, the effect of force on single-bond adhesion or kinetics can be studied. The lifetime of a single bond, which is indicative of a strong or weak adhesion and is the reciprocal of the reverse reaction rate constant, can be calculated by extrapolation if a correlation between the force and adhesion lifetime can be established experimentally. This requires that both receptor and ligand are anchored strongly on a surface (for example, via covalent bonds). However, if a receptor is anchored on a cell surface, the probability of uprooting it cannot be ignored, as demonstrated by Shao and Hochmuth in a study that involved multiple linkages with various strengths between a bead and a neutrophil (58). In that study, the adhesion lifetime actually represents how long it takes to uproot one of three receptors on a human neutrophil: Lselectin, CD18, and CD45. With the MAT, how accurately the adhesion lifetime can be measured depends upon the exposure time of the camera working with the micropipette-manipulation system. For an analog camera, the time resolution is around 0.03 second. Since it takes about 0.1 second to reverse the pressure from positive to negative in the manometer of the MAT (unpublished data), the measured adhesion lifetime should be at least a few tenths of a second. Otherwise, adhesion events will be masked by non-adhesion events.

One major issue that is involved in single-bond studies is whether a single bond is really present in an experiment. This is an issue complicated by many factors such as receptor valency, molecular clustering, and surface topography, as noted in the review by Zhu et al. (61). For uniformly-distributed molecules and monovalent bindings, it has been shown that, as long as the adhesion frequency is low (less than 25\%), adhesion is dominated mainly by single bonds $(58,62)$. For cell-bead or cell-substrate interactions, even in the presence of ligand clustering on the bead, our Monte Carlo simulation has shown that this criterion is still valid (unpublished data). Also, our simulation has shown that the single-bond percentage among all adhesion events will increase with ligand clustering if other conditions remain the same, as shown in Figure 5. However, whether this criterion still holds for more complex situations remains to be seen.

\section{CONCLUDING REMARKS}

We have presented a concise overview of micropipette-based techniques that can be used for quantifying cell-adhesion strength. Special attention was given to the MAT, which has an enormous potential for the application of femtonewton-scale forces. Much progress has been made and much insight has been gained with these micropipette-based techniques, yet their adoption has been restricted by the presently limited degree of automation in their setup and the time-consuming data analysis. Nevertheless, their versatility overcomes many of their shortcomings. Besides, they provide us several useful tools with some unique features for studying cellular and molecular biomechanics, especially quantifying celladhesion strength.

\section{ACKNOWLEDGEMENTS}

The authors gratefully acknowledge the support by the National Institutes of Health (R01 HL069947 and R21 RR017014). We thank Yong Chen, Baoyu Liu, and $\mathrm{Yan} \mathrm{Yu}$ for their assistance with analyzing and measuring bead motion in a glass tube. We also thank Dr. Salvatore Sutera for his critical reading of the manuscript.

\section{REFERENCES}

1. McClay, D. R., G. M. Wessel and R. B. Marchase: Intercellular recognition: quantitation of initial binding events. Proc Natl Acad Sci USA 78, 4975-9 (1981)

2. Cozens-Roberts, C., J. A. Quinn and D. A. Lauffenburger: Receptor-mediated adhesion phenomena: Model studies with the radial-flow detachment assay. Biophys J 58, 107-25 (1990)

3. Hochmuth, R. M., N. Mohandas and P. L. J. Blackshear: 
Measurement of the elastic modulus for red cell membrane using a fluid mechanical technique. Biophys $J$ 30, 747-62 (1973)

4. Weiss, L: The measurement of cell adhesion. Exp Cell Res 8, 141-53 (1961)

5. Evans, E. A: Minimum energy analysis of membrane deformation applied to pipet aspiration and surface adhesion of red blood cells. Biophys J 30, 265-84 (1980)

6. Binnig, G., C. F. Quate and C. Gerber: Atomic force microscope. Phys Rev Lett 56, 930-3 (1986)

7. Ashkin, A. and J. M. Dziedzic: Optical trapping and manipulation of viruses and bacteria. Science 235, 1517-20 (1987)

8. Kamimura, S. and K. Takahashi: Direct measurement of the force of microtubule sliding in flagella. Nature 293, 566-8 (1981)

9. Smith, S. B., L. Finzi and C. Bustamante: Direct mechanical measurement of the elasticity of single DNA molecules by using magnetic beads. Science 258, 1122-6 (1992)

10. Evans, E., K. Ritchie and R. Merkel: Sensitive force technique to probe molecular adhesion and structural linkages at biological interfaces. Biophys $J$ 68, 2580-7 (1995)

11. Shao, J.-Y. and R. M. Hochmuth: Micropipette suction for measuring piconewton forces of adhesion and tether formation from neutrophil membranes. Biophys J 71, 2892901 (1996)

12. Mitchison, J. M. and M. M. Swann: The mechanical properties of the cell surface: I. the cell elastimeter. $J$ Exp Biol 31, 443-60 (1954)

13. Mitchison, J. M. and M. M. Swann: The mechanical properties of the cell surface: II. the unfertilized sea-urchin egg. J Exp Biol 31, 461-72 (1954)

14. Rand, R. P. and A. C. Burton: Mechanical properties of the red cell membrane. I. Membrane stiffness and intracellular pressure. Biophys J 4, 115-35 (1964)

15. Rand, R. P: Mechanical properties of the red cell membrane. II. Viscoelastic breakdown of the membrane. Biophys J 17, 303-16 (1964)

16. Evans, E., R. E. Waugh and L. Melnik: Elastic area compressibility modulus of red cell membrane. Biophys $J$ 216, 585-95 (1976)

17. Sung, K. L., L. A. Sung, M. Crimmins, S. J. Burakoff and S. Chien: Determination of junction avidity of cytolytic T cell and target cell. Science 234, 1405-8 (1986)

18. Waugh, R. E: Surface viscosity measurements from large bilayer vesicle tether formation. I. Analysis. Biophys J38, 19-27 (1982)

19. Hochmuth, R. M. and E. A. Evans: Extensional flow of erythrocyte membrane from cell body to elastic tether. I. Analysis. Biophys J 39, 71-81 (1982)

20. Sato, M., M. J. Levesque and R. M. Nerem: An application of the micropipette technique to the measurement of the mechanical properties of cultured bovine aortic endothelial cells. J Biomech Eng 109, 27-34 (1987)

21. Mohandas, N. and E. Evans: Mechanical properties of the red cell membrane in relation to molecular structure and genetic defects. Annu Rev Biophys Biomol Struct 23, 787818 (1994)

22. Waugh, R. E., R. M. Hochmuth: Mechanics and deformability of hematocytes. In: The Biomedical Engineering Handbook. Ed: Bronzino JD, Boca Raton, FL, CRC Press, Inc., 474-86, (1995)

23. Hochmuth, R. M: Micropipette aspiration of living cells. J Biomech 33, 15-22 (2000)

24. Chesla, S. E., P. Selvaraj and C. Zhu: Measuring twodimensional receptor-ligand binding kinetics by micropipette. Biophys J 75, 1553-72 (1998)

25. You, J., A. M. Mastro and C. Dong: Application of the dual-micropipet technique to the measurement of tumor cell locomotion. Exp Cell Res 248, 160-71 (1999)

26. Dong, C., M. J. Slattery, B. M. Rank and J. You: In vitro characterization and micromechanics of tumor cell chemotactic protrusion, locomotion, and extravasation. Ann Biomed Eng 30, 344-55 (2002)

27. Shao, J.-Y. and J. Xu: A modified micropipette aspiration technique and its application to tether formation from human neutrophils. J Biomech Eng 124, 388-96 (2002)

28. Tozeren, A., K. L. Sung and S. Chien: Theoretical and experimental studies on cross-bridge migration during cell disaggregation. Biophys J 55, 479-87 (1989)

29. Sung, K. L., E. Saldivar and L. Phillips: Interleukin-1 beta induces differential adhesiveness on human endothelial cell surfaces. Biochem Biophys Res Commun 202, 866-72 (1994)

30. Tozeren, A., L. H. Mackie, M. B. Lawrence, P. Y. Chan, M. L. Dustin and T. A. Springer: Micromanipulation of adhesion of phorbol 12-myristate-13-acetate-stimulated $\mathrm{T}$ lymphocytes to planar membranes containing intercellular adhesion molecule-1. Biophys $J$ 63, 247-58 (1992)

31. Tözeren, A., K.-L. P. Sung, L. A. Sung, M. L. Dustin, P.Y. Chan, T. A. Springer and S. Chien: Micromanipulation of 
adhesion of a jurkat cell to a planar bilayer membrane containing lymphocyte function-associated antigen 3 molecules. J Cell Biol 116, 997-1006 (1992)

32. Sung, K. L., L. L. Steele, D. Whittermore, J. Hagan and W. H. Akeson: Adhesiveness of human ligament fibroblasts to laminin. J Orthop Res 13, 166-73 (1995)

33. Sung, K. L., L. Yang, D. E. Whittemore, Y. Shi, G. Jin, A. H. Hsieh, W. H. Akeson and L. A. Sung: The differential adhesion forces of anterior cruciate and medial collateral ligament fibroblasts: effects of tropomodulin, talin, vinculin, and alpha-actinin. Proc Natl Acad Sci USA 93, 9182-7 (1996)

34. Evans, E., R. Merkel, K. Ritchie, S. Tha and A. Zilker: Picoforce method to probe submicroscopic actions in biomembrane adhesion. In: Methods for studying cell adhesion. Eds: Bongrand P, Claesson PM and Curtis AS, 123-37, Berlin, Springer-Verlag (1994)

35. Evans, E: Looking inside molecular bonds at biological interfaces with dynamic force spectroscopy. Biophys Chem 82, 83-97 (1999)

36. Evans, E: Energy landscapes of biomolecular adhesion and receptor anchoring at interfaces explored with dynamic force spectroscopy. Faraday Discuss 111, $1-16(1998)$

37. Evans, E., A. Leung, D. Hammer and S. Simon: Chemically distinct transition states govern rapid dissociation of single L-selectin bonds under force. Proc Natl Acad Sci USA 98, 3784-9 (2001)

38. Evans, E., V. Heinrich, F. Ludwig and W. Rawicz. Dynamic tension spectroscopy and strength of biomembranes. Biophys J 85, 2342-50 (2003)

39. Evans, E. and K. Ritchie: Dynamic strength of molecular adhesion bonds. Biophys J 72, 1541-55 (1997)

40. Evans, E: Looking inside molecular bonds at biological interfaces with dynamic force spectroscopy. Biophys Chem 82, 83-97 (1999)

41. Evans, E. and K. Ritchie: Strength of a weak bond connecting flexible polymer chains. Biophys $J$ 76, 2439-47 (1999)

42. Evans, E. and F. Ludwig: Dynamic strengths of molecular anchoring and material cohesion in fluid biomembranes. J Phys -Condens Matter 12, 8A, A315A320 (2000)

43. Evans, E., A Leung, D. Hammer and S. Simon: Chemically distinct transition states govern rapid dissociation of single L-selectin bonds under force. Proc Natl Acad Sci USA 98, 3784-9 (2001)

44. Evans, E: Probing the relation between force--lifetime-and chemistry in single molecular bonds. Annu Rev
Biophys Biomol Struct 30, 105-28 (2001)

45. Shao, J.-Y: Finite element analysis of imposing femtonewton forces with micropipette manipulation. Ann Biomed Eng 30, 546-54 (2002)

46. Shao, J.-Y., H. P. Ting-Beall and R. M. Hochmuth: Static and dynamic lengths of neutrophil microvilli. Proc Natl Acad Sci USA 95, 6797-802 (1998)

47. Gelles, J., B. J. Schnapp and M. P. Sheetz: Tracking kinesin-driven movements with nanometer-scale precision. Nature 331, 450-3 (1988)

48. Cox, R. G. and S. G. Mason: Suspended particles in fluid flow through tubes. Annual Review of Fluid Mechanics 3, 291-316 (1971)

49. Ota, T., T. Sugiura and S. Kawata: Surface-force measurement with a laser-trapped microprobe in solution. Applied Physics Letters 80, 3448-50 (2002)

50. Levin, J. D., H. P. Ting-Beall and R. M. Hochmuth: Correlating the kinetics of cytokine-induced E-selectin adhesion and expression on endothelial cells. Biophys $J 80$, 656-67 (2001)

51. Dai, J. and M. P. Sheetz: Mechanical properties of neuronal growth cone membrane studied by tether formation with laser optical tweezers. Biophys $J$ 68, 988-96 (1995)

52. Marcus, W. D. and R. M. Hochmuth: Experimental studies of membrane tethers formed from human neutrophils. Ann Biomed Eng 30, 1273-80 (2002)

53. Evans, E. and A. Yeung: Hidden dynamics in rapid changes of bilayer shape. Chem Phys Lipids 73, 39-56 (1994)

54. Evans, E., Bowman, Howard, Leung, Andrew, Needham, David, Tirrell and David: Biomembrane templates for nanoscale conduits and networks. Science 273, 933-935 (1996)

55. Hochmuth, R. M., J.-Y. Shao, J. Dai and M. P. Sheetz: Deformation and flow of membrane into tethers extracted from neuronal growth cones. Biophys $J 70,358-$ 69 (1996)

56. Waugh, R. E: Surface viscosity measurements from large bilayer vesicle tether formation: II. Experiments. Biophys J 38, 29-37 (1982)

57. Hochmuth, R. M. and W. D. Marcus: Membrane tethers formed from blood cells with available area and determination of their adhesion energy. Biophys $J$ 82, 29649 (2002)

58. Shao, J.-Y. and R. M. Hochmuth: Mechanical anchoring strength of L-selectin, $\beta_{2}$ integrins and CD45 to neutrophil cytoskeleton and membrane. Biophys $J 77,587-$ 


\section{Quantification of Cell-Adhesion Strength}

$96(1999)$

59. Spillmann, C., D. Osorio and R. Waugh: Integrin activation by divalent ions affects neutrophil homotypic adhesion. Ann Biomed Eng 30, 1002-11 (2002)

60. Lomakina, E. B. and R. E. Waugh: Micromechanical Tests of Adhesion Dynamics between Neutrophils and Immobilized ICAM-1. Biophys J 86, 1223-33 (2004)

61. Zhu, C., M. Long, S. E. Chesla and P. Bongrand: Measuring receptor/ligand interaction at the single-bond level: experimental and interpretative issues. Ann Biomed Eng 30, 305-14 (2002)

62. Piper, J. W., R. A. Swerlick and C. Zhu: Determining force dependence of two-dimensional receptor-ligand binding affinity by centrifugation. Biophys $J$ 74, 492-513 (1998)

Key Words: Cell biomechanics, Receptor-ligand kinetics, Tether formation, Single bond, Receptor anchorage, Adhesion energy, Review

Send correspondence to: Jin-Yu Shao, Ph.D., Department of Biomedical Engineering, Washington University in St. Louis, CB 1097, Rm 290E Uncas A. Whitaker Hall, One Brookings Drive, St. Louis, MO 63130-4899, Tel: 314-9357467, Fax: 314-935-7448, E-mail: shao@biomed.wustl.edu 